\title{
Psychotherapeutic leadership and containment in psychiatry
}

\author{
Russell Gibson, Alex Till \& Gwen Adshead
}

\begin{abstract}
SUMMARY
Leadership is a key role for psychiatrists, with many models that can be adopted depending in part on the nature of the individual and their training, the primary task of the team and the nature of the organisation. In this article we suggest a new concept and model of leadership, 'psychotherapeutic leadership'. We discuss the theoretical background to this model, its benefits and the implications for training and professional development.
\end{abstract}

\section{LEARNING OBJECTIVES}

- Understand the concept and role of the psychiatrist in providing psychotherapeutic leadership at an individual patient, team and organisational level

- Explore how applying an understanding of psychodynamic principles in practice can provide containment in psychiatry and aid clinical leadership

- Understand the role of psychotherapeutically informed approaches in recognising factors influencing staff morale and burnout

\section{DECLARATION OF INTEREST}

None.

\section{KEYWORDS}

Education and training; personality disorders; ethics.

The role of medical leadership is of utmost importance in the delivery of high-quality healthcare services, especially in times of rising demand and financial austerity (Berwick 2013; Care Quality Commission 2017). The Francis report (Francis 2013) into the failings at Mid Staffordshire NHS Foundation Trust set a challenge for clinical leaders: deliver compassionate care while meeting the organisational demands of the National Health Service (NHS) (O'Sullivan 2014). All psychiatrists are expected to be leaders not just for themselves, but for their teams, their organisations and the systems they work within (Royal College of Psychiatrists 2010). Leadership should be a distinct and core component of a medical professional's training. There are many models of leadership that can be employed by psychiatrists and much depends on their individual nature, their training, the primary task of their team, and the nature or culture of their organisation and its systems.

The NHS has seen a wide spectrum of leadership models embraced, reflecting changes in political and socioeconomic factors, methods of commissioning services and the prevailing leadership theory at the time. For example, the NHS has moved from a professionally led and delivered service in which the degree of clinical autonomy maintained facilitated a more 'hierarchical' and 'heroic' model of leadership (King's Fund 2011) to onein which, partly in response to financial austerity and rising demand, commissioners 'purchase' services on behalf of the consumers i.e. patients. This more commercial model is radically different from the early traditions in medicine and, combined with the latest thoughts on leadership, has led to a more collective (West 2014) or distributed (Storey 2013) model of leadership throughout healthcare where leadership at all levels of the organisation is recognised and developed.

Within mental health services specifically there has been a dramatic change over the past 30 years. Traditional clinical models based on stigmatising long-stay residential care and assumed incapacity of psychiatric patients has progressed to a model that emphasises community-based recovery, self-management and parity of esteem (South London and Maudsley NHS Foundation Trust 2010; King's Fund 2015). An emphasis on recovery and rehabilitation has led to thousands of people with psychiatric diagnoses feeling more empowered and able to express their own views about their experience as patients and become involved in design of services (Donetto 2014). There is now wider availability of evidence-based psychological therapies for different levels of need, and national guidelines on the psychological therapies for a range of different conditions, including disorders such as personality disorder and psychosis.

However, while generalised outcomes, rapid recovery and discharge from services are seen as the ideal, it could be argued that a culture of 'anti-dependence' has been fostered, in which there is 'no real compassion for vulnerability' (Menon 2015) and non-medical professionals deliver manual-based short-term therapies at lesser cost.
Russell Gibson BSc (Hons) MBBCh MSc MRCPsych is a psychiatric core trainee, soon to enter specialty dual general adult and old age training. He is an Academic Clinical Fellow in

Psychiatry at Exeter University with an interest in clinical leadership, quality improvement and teaching. Alex Till is a psychiatric core trainee and vice-chair of the Royal College of Psychiatrists' Psychiatric Trainees' Committee. He is an Honorary Clinical Senior Lecturer at Swansea University and former National Medical Director's Clinical Fellow 2016-2017. Gwen Adshead is a forensic psychiatrist and group psychotherapist with an interest in attachment theory and dynamics in organisations. She has worked in high and medium secure services and has an active interest in ethics in mental healthcare.

Correspondence Dr Russell Gibson, College House, St Luke's Campus, Magdalen Road, Exeter EX1 2LU, UK. Email: russell.gibson@nhs.net

Copyright and usage (C) The Royal College of Psychiatrists 2018 
Medical psychotherapy has all but vanished in the NHS as the general psychiatrist's roles and responsibilities have arguably been pared back to providing consultation and advice for colleagues on complex and risky cases, with limited direct patient contact and an emphasis on prescribing psychotropic medications and risk management, as outlined in the Department of Health's New Ways of Working reports (Department of Health $2005,2010)$. This change means that psychiatrists have more of a supervising role, while other professions such as psychologists, mental health nurses and social workers assume broader and arguably more advanced roles in delivering direct patient care (Katschnig 2010). This raises concerns, because if psychiatrists do not continue to see patients on the 'front line' and see only a small number of cases while supervising others who are seeing vastly more, it is perhaps 'only a matter of time before we lose respect, credibility and competence' (Gee 2007).

Continuing to neglect the vital role of the psychiatrist, particularly in providing psychotherapeutic care, will hinder the development of psychologically informed clinicians and have serious consequences not just for patient care but for psychiatrists' ability to provide psychotherapeutic leadership for their teams, their organisations and the systems they work within and often lead (Allison 2016; Cammell 2016).

In this article we review psychiatrists' rolein providing medical leadership and combine previous discussions of how psychiatrists face unique leadership challenges with fresh psychotherapeutically informed perspectives to define the concept and model of 'psychotherapeutic leadership'. We explore how to develop and implement this leadership model, the positive impact it can have on individual patient care, the multidisciplinary team, the organisation and the wider system, alongside the risks and negative impact it can have on individual psychiatrists without the proper training and organisational support.

\section{Developing psychotherapeutic leadership}

Leadership can and should be learnt. Although this learning must be strengthened at both an

BOX 1 Psychotherapeutic leadership: a definition

Psychotherapeutic leadership involves the incorporation of psychotherapeutic principles into leading individual patient care, mental health teams, organisations and the wider system within which they work. It offers a psychologically minded understanding of situations and provides emotional containment for patients and teams, through support at the individual level and through psychologically minded service design at the organisational level. undergraduate and postgraduate level in medical education, there are inherent components of a doctor's training and, in particular, psychiatric training that nurture their role as leaders in healthcare. Specifically, medical training enables individuals to think systemically and to go beyond the superficial presentation of a problem. Doctors learn not to accept without question what is said to them or take at face value the symptoms reported or signs elicited. Doctors are trained to think about what has not been said or cannot be seen; and to think about how strains in one human system can produce symptoms and signs in another. Doctors ideally learn to use a curious and inquisitive approach to asking people about experience; and they also learn how to contain patients' unusual and individually unique ideas, concerns and expectations about what might be wrong.

Psychiatric training in particular stimulates doctors to consider a holistic view of a patient's problem, allowing them to coordinate care across all domains of the biopsychosocial model of illness (Craddock 2010). Through their training psychiatrists learn to understand the underlying psychological basis for human interactions, how this can lead to maladaptive behaviour and how to apply psychotherapeutic theories and techniques to help understand and overcome problems (Royal College of Psychiatrists 2016). It helps them establish therapeutic alliances and teaches them how to contain and manage some of the most challenging patient presentations a doctor can encounter, how to contain their own anxieties when making positive risk-taking decisions with patients who are sometimes at very high risk of doing direct harm to themselves or others, and how group dynamics can be influenced to contain the anxieties of the multidisciplinary teams they work within.

This is why it takes so long to train a doctor and why psychiatrists are unique owing to the nuances in their training that affect the style of medical leadership they employ, which, we suggest, can be conceptualised as a new model of leadership, 'psychotherapeutic leadership' Box 1).

\section{Psychotherapeutic leadership for individual patients}

Whether physical or psychological, illness and pain stimulate innate attachment systems and lead people to seek care and containment of distress from identified caregivers (Solomon 1996; Hunter 2001). People in distress want and need a personalised relationship that reduces arousal and anxiety, sustains hope for recovery and is honest and transparent about the difficulties that may lie ahead. 
Containing distress through the combination of these qualities has been key to medical professionals since the time of Hippocrates and is essential to providing person-centred care. Having a named consultant that they know and can relate to is a crucial aspect of this and affects a patient's experience (Reed 1991). As now recommended (Academy of Medical Royal Colleges 2014), all patients admitted to an NHS hospital should have a named consultant or clinician. This is necessitated in mental healthcare by the requirement in England and Wales for a named 'responsible clinician', most often a consultant psychiatrist, for patients detained under the Mental Health Act 1983 (amended 2007), but it may also be particularly helpful for patients (such as those with personality disorder) whose problems have a strongly relational aspect or who defend against distress by using dysfunctional behaviours that keep psychological pain out of awareness. For these patients, a psychiatrist's medical training and ability to lead a person-centred psychotherapeutic approach, integrated into a biopsychosocial model of illness, is vital and it helps psychiatrists to accept that people's minds are complex and sometimes opaque, to emphasise relationships as a way of understanding mental function and to use what Daniel Kahneman (Kahneman 2011) calls 'system 2' thinking, which prioritises reflection and

BOX 2 Case vignette: a person-centred psychotherapeutic approach

Arthur is a 23-year-old man with a diagnosis of schizophrenia who has been seeing a psychiatrist ( $D r A$ ) regularly for the past 18 months. Dr A congratulates Arthur on his recent good progress towards recovery and tells him that the team feels that he can be discharged to a rehabilitation service. Arthur appears to agree with this plan; but a few weeks later, he starts to take drugs and to be rude to the staff. Some of the team express the view that Arthur does not really have schizophrenia but has dissocial personality disorder instead (despite the fact that his history has none of the diagnostic features). Dr A looks at Arthur's history again, and reminds himself that fears of abandonment have been part of Arthur's story since his childhood. Dr A wonders whether Arthur unconsciously feels abandoned, but is unable to verbalise this. Dr A concludes that Arthur is 'acting out' his fears. He raises this in conversation with Arthur, who denies it, but agrees to meet with $\operatorname{Dr} A$ and the community team more frequently. The drug-taking and rude behaviour stop and Arthur starts to attend a recovery college and a Hearing Voices group. Dr A talks to the staff about Arthur's fears and supports them in encouraging Arthur to ask for help more appropriately. responsivity over instant reaction, i.e. a slower, more reflective response to human interaction.

Without a psychotherapeutic approach, formulations can become decontextualised and/or solely biological, potentially resulting in the iatrogenic prescription of ineffective and potentially harmful psychotropic regimes as the psychiatrist seeks to 'do something' in the face of distress and complex situations where multiple psychological and social factors are at play (Cammell 2016).

In Box $2,{ }^{a}$ an example sets out how the use of a psychotherapeutic approach can help patients like Arthur. Without a psychotherapeutic approach, Arthur's behaviour may be seen as evidence of wilful rule breaking. He might have incorrectly received a whole new diagnosis and management plan (including a psychotropic regime) based only on some recent behaviour and, crucially, staff's negative response to this. A psychotherapeutic approach is especially useful for complex cases where patients demonstrate comorbidity and allows for a temporal perspective so that the individual's past presentation and history are integrated with their current emotions, state of mind and pattern of relationships. Dr A can help the staff manage their ordinary negative reactions to Arthur and be both boundaried and empathic in their response by showing compassion and highlighting that, in the context of his psychopathology, what is seen on the surface is not all there is to Arthur's mind or experience.

It is psychiatric training that allows doctors to develop this psychotherapeutic leadership and form relationships that empower patients and professionals alike. They come to appreciate the importance of understanding relationships in complex situations where protocols and guidelines (although useful in acute situations with time-limited conditions) have less utility and become uniquely positioned to provide oversight for an individual patient's care that contains both the patient's and team's emotions in a person-centred manner.

\section{Psychotherapeutic leadership within teams}

Through their training, psychiatrists develop the psychologically minded understanding, awareness and ability to incorporate psychotherapeutic principles into their practice. This underpins psychotherapeutic leadership and makes them ideally suited to lead and direct increasingly integrated and multidisciplinary teams (Royal College of Psychiatrists 2010).

Although in theory the multidisciplinary nature of modern healthcare should distribute responsibility and create a sense of collective leadership among the professionals involved, the team often 'grants leadership', and therefore implicit responsibility, to the doctor (De Waal 2013). While this occurs in a The case vignettes in this article are fictitious. 
nearly all medical specialties, in England and Wales it is arguably most apparent in psychiatry because of the above-mentioned requirement for patients detained under the Metal Health Act to have an identified responsible clinician. Although this may be seen to exacerbate the hierarchy, doctors are in fact more likely than other healthcare professionals to be questioned and accept decisions contrary to their initial stance (Gair 2001).

It is vital that psychiatrists continue to be provided with the substrate (in training, support and ongoing direct patient care) to develop psychotherapeutically informed approaches that help them manage the attitudes and expectations of different professionals who may hold differing opinions (O'Sullivan 2014) and contain their own as well as the patient's and team's anxiety, as ultimately it is the psychiatrist, as the responsible clinician for a patient detained under the Mental Health Act, who is held individually accountable for the patient's care.

\section{Containment and emotional contagion}

Two key concepts that help psychiatrists adopt psychotherapeutic leadership are 'containment' and 'emotional contagion'.

BOX 3 Case vignette: a psychotherapeutic approach to team dynamics

Ben is a 34-year-old man with a diagnosis of emotionally unstable personality disorder, based on a long history of self-harming behaviour. He has been admitted to an inpatient psychiatric ward following an overdose of paracetamol. After several days on the ward and during a multidisciplinary team meeting, some staff report that he is pleasant and settled and willing to engage in a treatment plan. Others state that he has not improved, that he is difficult to work with and should be discharged immediately. Using a psychotherapeutic approach that considers both defences and developmental psychopathology, Ben's psychiatrist sees that Ben tends to be more confrontational with those he perceives as being in a position of authority (such as the male ward manager), but much less so with staff whom he does not see as being in charge or judging him (such as the female healthcare support workers). He is subconsciously mirroring similar interactions from his past relationships with his father and mother. The psychiatrist offers this hypothesis in the team meeting, and staff members agree that this may explain their different reactions to Ben. They try to ensure that the ward manager gets a chance to work collaboratively with Ben and that the healthcare support workers remind Ben of the boundaries on the ward and his role in a safe move into the community. The psychiatrist also talks to Ben about whether he may have mixed feelings about moving on from the ward, where he feels safe. Ben responds positively to this, and he agrees to a discharge plan that is successfully started the following week.
Bion's concept of 'containment' (Bion 1985) is important in terms of understanding how a caregiver (in this case the psychiatrist) processes the emotions of another person (the patient and the multidisciplinary team) who is dependent on them.

For example, containment can be observed in infancy. A baby does not yet have all the synaptic connections necessary to manage strong emotions such as fear or anger. A mother recognises this and, by holding the baby and demonstrating attunement to the painful emotions, she reduces the baby's anxiety and arousal. This reassures the baby that such affects are tolerable. However, the baby has only immature psychological defences to manage distress, so the mother also experiences the baby's feelings through a process of projection. Mothers therefore have to contain not only their baby's projected distress, but also their own emotional responses to the baby's distress; ideally, they will use mature psychological defences to manage these feelings, so they do not project back into the baby. Mothers who cannot do this get angry with their babies when the infants are distressed and may react with hostility. This can lead to an insecure attachment between baby and mother, which later in life could contribute to the development of enduring patterns of disturbed interpersonal relationships that are often present in people with borderline (or emotionally unstable) personality disorder (Agrawal 2004).

Patients whose capacity to manage distress is impaired tend to externalise their distress and use immature defences such as acting out, substance misuse, somatisation and displacement as a way of coping (Cramer 2000). In these situations, mental health professionals can be left trying to recognise, buffer and contain these emotions, projected beyond the patient. Freud called these reactions 'transference and countertransference' (Freud 1938), but these terms do not really do justice to the complexity of interpersonal emotions that can be experienced between people who are working together in situations where there is high expressed emotion, such as that often found in mental healthcare.

The second key concept in psychotherapeutic leadership is that of the 'emotional contagion' in which group members will quickly respond to the emotional state of others, often either mirroring these states or reacting against them (Barsade 2002).

Understanding the range of emotions felt by staff in relation to the experiences of patients with difficult or complex emotions, and leading them through it, is a vital feature of a psychiatrist's ability to display psychotherapeutic leadership (Box 3). Through this approach they are able to provide containment for staff by taking ownership of decisions when required, 
are inclusive in their interactions by embracing a collective leadership style, and enable psychological safety through a deeper understanding of team dynamics and through the facilitation of reflective practice with multidisciplinary team members.

In the example shown in Box 3, the psychiatrist recognises how the team is containing different aspects of the patient's emotional experience in a defence called 'splitting'. Team members are holding both aspects of what the patient feels, but also holding different responses to the patient; and these feelings are accompanied by cognitive distortions, such as 'black and white' thinking and jumping to conclusions. The psychiatrist takes note of the different responses; how the patient elicits sympathy from some staff and hostility from others. He also notes that these different reactions may reflect different aspects of the patient's mind; in this case, real ambivalence about whether he is safe enough in himself to leave the ward.

Alongside the regular ward business and action meetings, the psychiatrist employs psychotherapeutic leadership principles to ensure that reflective-practice meetings are integrated into the ward regime to allow for this type of discussion, where good-quality thinking about patients and their relationships with staff are facilitated. Not only does this improve therapeutic alliances with patients; as Hinshelwood \& Skogsted (2002) observe, these discussions help staff overcome immature psychological defence mechanisms when making difficult decisions and help promote team cohesiveness to ensure that everyone is working towards a common goal, especially when working with patients with complex needs, who can arouse strong emotions in staff.

The European Psychiatric Association guidance on the role and responsibilities of psychiatrists specifically highlights that 'one of the major expectations of the psychiatrist is their ability to tolerate high levels of anxiety in situations of considerable uncertainty while holding the team's anxiety and holding the hope for the patient so that the patient can continue to work through their personal and social emotional distress' (Bhugra 2015). Containing uncertainty as well as the anxiety of the patient and the clinical team is therefore a fundamental role expected of the psychiatrist. This can only be developed through training, reflective practice and experience in delivering psychotherapeutic care to patients.

\section{Emotional intelligence}

For psychiatrists to be most effective in their role as psychotherapeutic leaders, they must be able to gauge and respond to the emotional and social 'temperature' and 'mood' of situations, and to create an atmosphere that can contain the emotions of staff and allow people to express their emotions safely (O'Sullivan 2014). This requires the psychiatrist to develop a high emotional intelligence incorporating the multidimensional qualities of self-awareness, self-regulation, self-motivation, social awareness and social skills (Goleman 1998, 2017). This is again where psychiatric training comes to the fore: through regular psychologically informed practice and reflection, psychiatrists develop a greater understanding of interpersonal interactions between not just themselves and their patients, but also among their multidisciplinary colleagues, so that in the face of emotionally charged situations they are able to use these emotions positively to negotiate, build rapport and move otherwise 'stuck' situations forward (Iszatt-White 2009; Johnson 2013).

Adopting a psychodynamic approach to leadership within teams and organisations can allow leaders to identify the unconscious behaviours and patterns that may play out in 'organisational life' and are especially relevant to the role of leader as 'coach' (Ward 2014). Combined with their knowledge and experience in a variety of psychotherapeutic models, psychiatrists have an advantage that can help them to interpret and negotiate difficult and challenging interpersonal and situational conflicts in both team and organisational settings.

\section{Psychotherapeutic leadership in organisations}

In response to the Francis report (Francis 2013), Don Berwick, cofounder of the Institute for Healthcare Improvement, stated that healthcare leaders must embody the vision for high-quality care (Berwick 2013). Within mental healthcare, it is essential this vision is aligned with a psychotherapeutic approach, and psychiatrists have a collective responsibility to ensure that psychotherapeutic leadership exists throughout organisations in clinical, administrative and academic settings (Cammell 2016).

Psychiatrists are often ideally suited to bridging the divide between clinicians and managers of organisations and have developed many of the skills to become good or even 'great' medical leaders (Johnson 2013; Goodall 2016; Perry 2016). This is not just by virtue of their training as medical professionals; by applying their expert knowledge of psychiatric practice and group dynamics to organisational strategy psychiatrists can advocate for a 'psychotherapeutically informed' model of care for patients. They can also influence the organisation's culture to provide a 'just culture' with psychological safety, better working conditions and fairness to staff that will help to contain the anxiety of professionals 
and stimulate organisational learning (Dekker 2016; Goodall 2016).

By drawing on an enhanced understanding of object relations theory and the reciprocal roles developed in early life, and their relevance to the leaderfollower relationship, psychiatrists are assisted in reflecting on others' behaviours and the interrelationship with their own leadership style (Plakiotis 2017). By participating in regular Balint groups throughout training, psychiatrists develop a sense of self separate from the patient (Graham 2009) that can also enhance their ability to reflect on interactions with other healthcare professionals, managers and senior executives.

\section{Principles in practice: Project Air and the CHANGE Programme}

Project Air is an intervention developed in Australia specifically to improve how general mental health services manage and treat people with personality disorder, recognising the high cost to health services and the negative countertransference often elicited in staff members by this patient population (Project Air Strategy for Personality Disorders 2015). The project is evidence-based, utilises routine outcome measures and directly applies an understanding of psychotherapeutic principles to service delivery (Grenyer 2014). It has resulted in a number of positive outcomes, including reduced emergency department attendance, admissions and length of stay for people with personality disorder (Grenyer 2014). The combination of senior clinical leaders, including psychiatrists, overseeing implementation of the programme in a manner consistent with psychotherapeutic principles, allied with experienced clinical leadership for front-line staff, demonstrates

BOX 4 Psychotherapeutically informed service design: the CHANGE Programme

The CHANGE Programme developed within Devon Partnership NHS Trust is based on the structured clinical management approach for patients with emotionally unstable personality disorder. The programme is delivered over eight weekly hour-long sessions by the same trained professional, who is also the patient's designated recovery coordinator. Each weekly session is structured, including activities such as learning distress tolerance techniques and education about the person's diagnosis and treatment. The recovery coordinators are supervised by a psychologist on a weekly basis to discuss progress and any issues or challenges that may arise. At the end of the 8-week programme the patient can be referred on for further specialist psychological intervention if deemed appropriate.

(Devon Partnership NSH Trust 2017) a psychotherapeutic approach at a strategic level in an organisation. This is done through providing education and supervision to front-line clinicians, as well as improved access to therapeutic interventions and collaborative working between different professionals.

In the UK, the CHANGE Programme developed within Devon Partnership NHS Trust (Box 4) is another example of streamlining and directing resources to structured therapeutic interventions. Psychiatrists, along with other multidisciplinary team members, including managers, senior nursing staff and psychologists, have been involved in the conception, design and delivery of this service. By attending to relational aspects fundamental in delivering care for patients with personality disorder, such as consistent contact with the same member of staff, the CHANGE Programme demonstrates key aspects of psychotherapeutically informed care. Psychiatrists, who hold an overview of patient care, can therefore play an essential role in providing psychotherapeutic leadership and shaping organisational strategy and service design to provide psychotherapeutically designed services.

\section{Psychotherapeutic leadership and protecting against burnout}

\section{Protecting the team}

Burnout has been described as a combination of emotional fatigue, depersonalisation and lack of personal fulfilment (Maslach 1982). It has a negative impact on staff, patient safety and patient satisfaction (Vahey 2004; Hall 2016). When staff work within a poor organisational culture or where there is weak cohesion within teams, they are likely to suffer higher rates of burnout and offer inadequate care to their patients (Riordan 1999; Lasalvia 2009; Morse 2012).

In the UK, mental health services face financial austerity, increasing demand and a workforce crisis (Royal College of Psychiatrists 2009; King's Fund 2015; Lovett 2017). Combined with the continuing drive to provide high-quality care, increasing pressure on clinical teams is, unsurprisingly, contributing to stress and burnout among staff (Imo 2017). However, it has also been suggested that lack of reward for care and an organisational climate that supports unfairness may have a greater effect on burnout among mental health staff than even high case-loads; therefore leadership that can address these issues can help to provide some form of containment for staff (Lasalvia 2009; Green 2014).

Dealing with extreme emotional distress and chronic psychiatric illness is draining for mental healthcare professionals, sapping their energy and 
limiting their capacity to work in difficult clinical situations. Adopting psychotherapeutically informed leadership during service design, as described in Box 5 , is therefore particularly important; not only can it provide added insight into patients' behaviour, enabling effective person-centred care, but also it can help front-line staff to safely express their feelings, learn from them, and manage their own emotional responses to protect against burnout and build psychological resilience. In so doing, staff members are motivated and encouraged to utilise psychotherapeutic principles that could otherwise be neglected, to the detriment of patients and at the risk of increased staff burnout.

\section{Protecting the leader}

Although psychiatrists providing psychotherapeutic leadership can improve services and act as a protective factor against burnout in the multidisciplinary team, it is not without risks to the individual, who often contains the emotions of the team and, as responsible clinician, holds accountability for risk. As many as two-thirds of psychiatrists have been found to be experiencing high levels of emotional exhaustion (Kumar 2007) and they have long been identified as being at higher risk of burnout in comparison with colleagues in other medical specialties and with other professionals working in mental health settings (Deary 1996; Lasalvia 2009). Patient suicide is one personal stress that many psychiatrists find it difficult to cope with (Fothergill 2004). Ensuring access to peer support and reflective practice groups can help clinicians manage emotional stressors and help move away from individual isolation to a culture of shared responsibility for clinician well-being and patient safety (Shapiro 2016).

Psychiatrists should be supported in developing their emotional intelligence as mitigation against

\section{BOX 5 Supporting staff improves patient care}

In response to the challenges of dealing with offenders with personality disorder, the National Offender Management Service in the UK has encouraged practitioners to take an attachment-based approach in which a single case manager is assigned to individuals throughout the entire criminal justice process. Practitioners are also trained to recognise the consequences of insecure or poo attachment, along with experiences of trauma, and how those with personality disorder interpret the thoughts and feelings of others, manage relationships and tend to have strong, explosive emotional outbursts. The practitioners are then supported by active encouragement to seek and engage with psychologically informed supervision and support.

(National Offender Management Service 2015) burnout (Weng 2011), but this does not remove the need for cultural changes to ensure that psychotherapeutic leadership is embodied at an organisational level. This will allow psychiatrists to manage their emotions and speak out about concerns in a psychologically safe environment where their anxieties can be contained and the burden of responsibility is distributed among the clinical team and throughout the organisation via the understanding and implementation of a just culture (Lasalvia 2009; Dekker 2016). If the approach is not embodied organisationally, it may lead to more burnout in individual clinicians, not less.

\section{Conclusions}

With the increasing drive to promote doctors into leadership positions throughout the NHS, we suggest that training psychiatrists to deliver psychotherapeutic leadership is essential. We argue that, for psychiatrists to deliver psychotherapeutic leadership, they must continue to have direct patient contact. They should have adequate training in psychotherapy, as well as an understanding and experience of applying psychotherapeutic principles in individual patient care, team dynamics and service design. They should also work in an open culture, where staff members are free to speak out about their anxieties and concerns, and are supported to access peer support, reflective practice and develop their own emotional intelligence.

Whether in the community or in in-patient services, psychotherapeutic leadership enables psychiatrists to lead on: understanding patients in more complex ways; containing their own and their team's emotions in relation to managing patients with challenging problems; and developing safer services with a psychotherapeutically informed model of person-centred care that embodies the psychological safety of a just culture. Further, if this approach goes beyond the individual and is embodied at an organisational level, it may lead to reductions in burnout and improved patient care.

\section{References}

Academy of Medical Royal Colleges (2014) Guidance for Taking Responsibility: Accountable Clinicians and Informed Patients. Academy of Medical Royal Colleges.

Agrawal HR, Gunderson J, Holmes BM, et al (2004) Attachment studies with borderline patients: a review. Harvard Review of Psychiatry, 12 : 94-104.

Allison S, Goodall A, Bastiampillai T (2016) Expert leadership - why psychiatrists should lead mental health services. Australas Psychiatry, 24: 225-7.
MCO answers

$1 \mathrm{~d} 2$ e 3 e 4 d 5 b 
Barsade SG (2002) The ripple effect: emotional contagion and its influence on group behavior. Administrative Science Quarterly, 47: 644-75.

Berwick D (2013) A Promise to Learn - A Commitment to Act: Improving the Safety of Patients in England. National Advisory Group on the Safety of Patients in England.

Bhugra D, Ventriglio A, Kuzman MR, et al (2015) EPA guidance on the role and responsibilities of psychiatrists. European Psychiatry, 30 417-22

Bion WR (1985) Container and contained. Group Relations Reader, 2(8): 127-33

Cammell P, Amos J, Baigent M (2016) How can psychiatrists offer psychotherapeutic leadership in the public sector? Australasian Psychiatry, 24 246-8.

Care Quality Commission (2017) The State of Care in Acute NHS Hospitals. Care Quality Commission.

Craddock N, Kerr M, Thapar A (2010) What is the core expertise of the psychiatrist? Psychiatrist, 34: 457-60.

Cramer $\mathrm{P}$ (2000) Defense mechanisms in psychology today: further processes for adaptation. American Psychologist, 55: 637-46.

De Waal H (2013) Clinical leadership. In Leadership in Psychiatry (ed D Bhugra, P Ruiz, S Gupta): 74-88. Wiley Blackwell.

Deary IJ, Agius R, Sadler A (1996) Personality and stress in consultant psychiatrists. International Journal of Social Psychiatry, 42 $112-23$

Dekker SW, Breakey H (2016) 'Just culture:' improving safety by achieving substantive, procedural and restorative justice. Safety Science, 85: 187-93. Department of Health (2005) New Ways of Working for Psychiatrists: Enhancing Effective, Person-Centred Services through New Ways of Working in Multidisciplinary and Multi-Agency Contexts. Department of Health.

Department of Health (2010) Responsibility and Accountability: Moving on for New Ways of Working to a Creative, Capable Workforce. Best Practice Guidance. Department of Health.

Devon Partnership NHS Trust (2017) The CHANGE Programme - What Does It Mean for Me? Information for People Using Our Services. Devon Partnership NHS Trust (https://www.dpt.nhs.uk/download/ yTxPLkUn80).

Donetto S, Tsianakas V, Glenn R (2014) Using Experience-Based Codesign (EBCD) to Improve the Quality of Healthcare: Mapping Where We Are Now and Establishing Future Directions: Final Report. King's College London.

Fothergill A, Edwards D, Burnard P (2004) Stress, burnout, coping and stress management in psychiatrists: findings from a systematic review. International Journal of Social Psychiatry, 50: 54-65.

Francis R (2013) Report of the Mid Staffordshire NHS Foundation Trust Public Inquiry: Executive Summary. TSO (The Stationery Office)

Freud S (1938) The Basic Writings of Sigmund Freud. Modern Library.

Gair G, Hartley T (2001) Medical dominance in multi-disciplinary teamwork: a case study of discharge decision making in a geriatric assessment unit. Journal of Nursing Management, 9: 3-11.

Gee M (2007) New Ways of Working threatens the future of the psychiatric profession. Psychiatric Bulletin, 31: 315.

Goleman D (1998) The emotional intelligence of leaders. Leader to Leader, 10: $20-6$

Goleman D (2017) Leadership That Gets Results. Harvard Business Review Press.

Goodall AH (2016) A theory of expert leadership (TEL) in psychiatry. Australasian Psychiatry, 24: 231-4.

Graham S, Gask L, Swift G, et al (2009) Balint-style case discussion groups in psychiatric training: an evaluation. Academic Psychiatry, 33: 198-203.

Green AE, Albanese BJ, Shapiro NM, et al (2014) The roles of individual and organizational factors in burnout among community-based mental health service providers. Psychological Services, 11: 41-9.
Grenyer B (2014) An integrative relational step-down model of care: the Project Air Strategy for Personality Disorders. ACPARIAN (Australian Clinical Psychologist), (9): 8-13.

Hall LH, Johnson J, Watt I, Tsipa A, O'Connor DB (2016) Healthcare staff wellbeing, burnout, and patient safety: a systematic review. PLoS One, 8 , 11: e0159015.

Hinshelwood RD, Skogstad W (2002) Observing Organisations: Anxiety, Defence and Culture in Health Care. Routledge.

Hunter J, Maunder RG (2001) Using attachment theory to understand illness behavior. General Hospital Psychiatry, 23: 177-82.

Imo UO (2017) Burnout and psychiatric morbidity among doctors in the UK: a systematic literature review of prevalence and associated factors. BJPsych Bulletin, 41: 197-204.

Iszatt-White M (2009) Leadership as emotional labour: the effortful accomplishment of valuing practices. Leadership, 5: 447-67.

Johnson JM, Stern TA (2013) Preparing psychiatrists for leadership roles in healthcare. Academic Psychiatry, 37: 297-300.

Kahneman D (2011) Thinking, Fast and Slow. Penguin.

Katschnig H (2010) Are psychiatrists an endangered species? Observations on internal and external challenges to the profession. World Psychiatry, 9: 21-8

King's Fund (2011) The Future of Leadership and Management in the NHS: No More Heroes. The King's Fund.

King's Fund (2015) Briefing: Mental Health Under Pressure. The King's Fund.

Kumar S, Fischer J, Robinson E, et al (2007) Burnout and job satisfaction in New Zealand psychiatrists: a national study. International Journal of Social Psychiatry, 53: 306-16

Lasalvia A, Bonetto C, Bertani M, et al (2009) Influence of perceived organisational factors on job burnout: survey of community mental health staff. British Journal of Psychiatry, 195: 537-44.

Lovett K (2017) Increasing the number of mental health staff must be a priority. Health Service Journal, 7 July.

Maslach C (1982) Burnout: The Cost of Caring. Prentice-Hall.

Menon A, Flannigan C, Tacchi M, et al (2015) Burnout - or heartburn? A psychoanalytic view on staff burnout in the context of service transformation in a crisis service in Leeds. Psychoanalytic Psychotherapy, 29: 330-42.

Morse G, Salyers MP, Rollins A, et al (2012) Burnout in mental health services: a review of the problem and its remediation. Administration and Policy in Mental Health, 39: 341-52.

National Offender Management Service (2015) The Offender Personality Disorder Pathway Strategy 2015 (Gateway Reference 04272). National Offender Management Service/NHS England.

O'Sullivan H, McKimm J (2014) The role of emotion in effective clinical leadership and compassionate care. British Journal of Hospital Medicine, 75: 281-6.

Perry J, Mason FL (2016) The value of psychiatrists in leadership and management. BJPsych Advances, 22: 263-8.

Plakiotis C (2017) Psychodynamic leadership approach and leader-member exchange (LMX): a psychiatric perspective on two leadership theories and implications for training future psychiatrist leaders. In GeNeDis 2016 (ed P Vlamos): 97-111 (Advances in Experimental Medicine and Biology, vol 988). Springer

Project Air Strategy for Personality Disorders (2015) Treatment Guidelines for Personality Disorders (2nd edn). NSW Health, Illawarra Health and Medical Research Institute.

Reed J (1991) The future for psychiatry. Psychiatric Bulletin, 15: 396-401.

Riordan C, Weatherly E (1999) Defining and measuring employees' identification with their work groups. Educational and Psychological Measurement, 59: 310-24.

Royal College of Psychiatrists (2009) Mental Health and the Economic Downturn: National Priorities and NHS Solutions (Occasional Paper OP70). Royal College of Psychiatrists. 
Royal College of Psychiatrists (2010) Role of the Consultant Psychiatrist: Leadership and Excellence in Mental Health Services (Occasional Paper OP74). Royal College of Psychiatrists.

Royal College of Psychiatrists (2016) CT1-3 Psychotherapy Trainee Guide. Royal College of Psychiatrists.

Shapiro J, Galowitz P (2016) Peer support for clinicians: a programmatic approach. Academic Medicine, 91(9): 1200-4.

Solomon J, George C (1996) Defining the caregiving system: toward a theory of caregiving. Infant Mental Health Journal, 17: 183-97.

South London and Maudsley NHS Foundation Trust, South West London and St George's Mental Health NHS Trust (2010) Recovery is for All: Hope, Agency and Opportunity in Psychiatry. A Position Statement by Consultant Psychiatrists. SLAM/SWLSTG.
Storey J, Holti R (2013) Towards a New Model of Leadership for the NHS. NHS Leadership Academy.

Vahey DC, Aiken LH, Sloane DM, et al (2004) Nurse burnout and patient satisfaction. Medical Care, 42(suppl 2): II57-66.

Ward G, Van de Loo E, ten Have S (2014) Psychodynamic group executive coaching: a literature review. International Journal of Evidence Based Coaching and Mentoring, 12(1): 63-78.

Weng HC, Hung CM, Liu YT, et al (2011) Associations between emotional intelligence and doctor burnout, job satisfaction and patient satisfaction. Medical Education, 45: 835-42.

West M, Eckert R, Steward K, et al (2014) Developing Collective Leadership for Healthcare. The King's Fund.
MCQs

Select the single best option for each question stem

1 Providing psychotherapeutic leadership:

a is solely the responsibility of the psychiatrist

b involves the psychiatrist containing all the anxiety of their colleagues

c negates providing structured interventions

d is applicable to individual patient care, clinical teams and organisations

e increases burnout among mental health professionals.

2 The advantages of psychotherapeutically informed care for the patient include reducing:

a excessive focus on biological formulation

b iatrogenic prescription of medication

c polypharmacy

d decontextualised formulation

e all of the above.
3 Psychiatrists can help provide psychotherapeutic leadership to clinical teams through:

a applying their knowledge and experience in psychotherapy

b their understanding of group dynamics

c providing containment of other staff member's anxiety

d employing their emotional intelligence to negotiate, build rapport and move forward

e all of the above.

4 Of the following professionals, those at highest risk of psychological burnout are:

a general practitioners

b psychiatric nurses

c psychologists

d psychiatrists

e social workers.
5 Actions to improve the implementation of psychotherapeutically informed patient care could include:

a reducing risk-taking in patient care

b providing psychological safety to speak out about concerns within a 'just culture'

c assigning all responsibility for risk assessment and management to the individual clinician

d preventing patient suicide

e promoting resilience in clinicians rather than systemic interventions. 